\title{
Generating an Educational Domain Checklist through an Adaptive Framework for Evaluating Educational Systems
}

\author{
Roobaea S. AlRoobaea \\ Faculty of Computing and \\ Information Systems, Taif \\ University, Saudi Arabia, \& School \\ of Computing Sciences, \\ University of East Anglia, UK
}

\author{
Ali H. Al-Badi \\ Department of Information Systems, \\ Sultan Qaboos University, Oman
}

\author{
Pam J. Mayhew \\ School of Computing Sciences \\ University of East Anglia, UK
}

\begin{abstract}
The growth of the Internet and related technologies has enabled the development of a new breed of dynamic websites that is growing rapidly in use and that has had a huge impact on many businesses. One type of websites that have been widely spread and are being widely adopted is the educational websites. There are many forms of educational websites, such as free online websites and Web-based server software. This creates challenges regarding their continuing evaluation and monitoring in order to measure their efficiency and effectiveness, to assess user satisfaction and, ultimately, to improve their quality.
\end{abstract}

The lack of an adaptive usability checklist for improvement of the usability assessment process for educational systems represents a missing piece in 'usability testing'. This paper presents an adaptive Domain-Specific Inspection (DSI) checklist as a tool for evaluating the usability of educational systems. The results show that the adaptive educational usability checklist helped evaluators to facilitate the evaluation process. It also provides an opportunity for website owners to choose the usability area(s) that they think need to be evaluated. Moreover, this method was more efficient and effective than user testing (UT) and heuristics evaluation (HE) methods.

Keywords-Heuristic evaluation (HE);User Testing (UT);Domain Specific Inspection (DSI); Adaptive Framework; Adaptive Checklist

\section{INTRODUCTION}

It is clear that Heuristic Evaluation (HE) and User Testing (UT) are the most important traditional usability evaluation methods for ensuring system quality and usability (Lindgaard and Chattratichart, 2007). Currently, complex computer systems, mobile devices and their applications have made usability evaluation methods more critical; however, usability differs from one product to another depending on product characteristics. It is clear that users have become the most important factor impacting on the success of a product; if a product is produced and is then deemed not useful by the endusers, it is a failed product; nobody can use it and the company cannot make money (Nielsen, 2001). Nayebi et al., (2012) asserted, "Companies are endeavoring to understand both user and product, by investigating the interactions between them".

Traditional usability measures of effectiveness, efficiency and satisfaction are not adequate for the new contexts of use
(Zaharias and Poylymenakou, 2009). HE has been claimed to be too general and too vague for evaluating new products and domains with different goals; It can produce a large number of false positives, and it is unlikely to encompass all the usability attributes of user experience and design in modern interactive systems (Chattratichart and Lindgaard, 2008). UT has been claimed to be costly, time consuming, prone to missing consistency problems and subject toenvironmental factors (Oztekinet et al., 2010). To address these challenges, many frameworks and models have been published to update usability evaluation methods (UEMs) (Alias et al., 2013); however, these frameworks and models are not applicable to all domains because they were developed to deal with certain aspects of usability in certain areas (Coursaris and Kim, 2011).

The adaptive framework was originally constructed and then evaluated in both the educational domain and social networks domain to generate domain specific-context inspection (DSI) method; in those experiences, it delivered interesting results by discovering more real usability problems in specific usability areas than HE or UT (AlRoobaea et al., 2013a) (AlRoobaea et al., 2013b). An adaptive checklist based upon the DSI method for facilitating the educational evaluation process was developed. The main objective of this paper is to address the challenges that were raised and to present this checklist which can be applied to any system in the educational domain as a tool that can be used by designers, developers, instructors, and website owners to design an interactive interface or assess the quality of existing systems. It also allows anyone to adopt any area of usability or any principle to determine the usability problems related to the five specific areas in educational system.

This paper is organized in the following way. Section 2 starts with a brief literature review including a summary of the adaptive framework. Section 3 presents the adaptive DSI checklist. Section 4presents a discussion of the findings. Section 5 presents the conclusion and future work.

\section{LITERATURE REVIEW}

\section{A. Background and Motivation}

The primary concern of interaction design is to develop interactive products or technologies that are usable. This means 
that the products should be easy to learn, effective to use, and offering a pleasurable user experience. Basically, a website is a product, and the quality of a product takes a significant amount of time and effort to develop. Web design is a key factor in determining the success of any website, and users should be the priority in the designers' eyes because usability problems in a website can have serious ramifications, over and above failing to meet the users' needs (Chen and Macredie, 2005). A high-quality product is one that provides all the main functions in a clear format, and that offers good accessibility and a simple layout to avoid users spending more time learning how to use it than satisfying their needing; these are the fundamentals of the 'usability' of a product. Poor product usability may have a negative impact on various aspects of the organization, and may not allow users to achieve their goals efficiently, effectively and with a sufficient degree of satisfaction [ISO, 1998]. The website consultants and marketing sectors have understood the number of hits, customer return rate, and customer satisfaction are extremely affected by the usability of a website [ Rogers et al., 2007].

Designing interactive products and evaluating them are common stages of product development. However, the current traditional usability methods to measure effectiveness, efficiency and satisfaction are not adequate for the new contexts of use, and are not stable in the modern dynamic environment (Mankoff et al., 2003); Several studies have emphasized the importance of developing new kinds of usability evaluation methods and of constantly improving and making modifications to existing methods as a matter of priority, in order to increase their effectiveness (Guo et al., 2011). Having extensively reviewed the existing literature on web usability evaluation methods; this research is unique in systematically constructing an adaptive framework that is applicable across numerous domains. This DSI framework generates DSI checklist / tool for assessing and improving the usability of a product.

\section{B. Description of the Adaptive Framework}

The adaptive framework was developed according to an established methodology in HCI research (AlRoobaea et al., 2013a); (AlRoobaea et al., 2013b). It consists of four development steps as follows:

Development Step One (D1: Familiarization): This stage starts by justifying the need to develop a method that is specific, productive, useful, usable, reliable and valid, which can be used to evaluate an interface design in the chosen domain. It entails reviewing all the published material in the area of UEMs but with a specific focus on knowledge of the chosen domain. Also, it seeks to identify an approach that would support developers and designers in thinking about their design from the intended end-users' perspective.

Development Step Two (D2: User Input): This stage consists of mini-user testing (task scenarios, think aloud protocol and questionnaire). Users are asked to perform a set of tasks on a typical domain website and then asked to fill out a questionnaire. The broad aim of this stage is to elicit feedback on a typical system from real users in order to appreciate the user perspective, to identify requirements and expectations and to learn from their errors. Understanding user needs has long been a key part of user design, and so this step directly benefits from including the advantages of user testing.

Development Step Three (D3: Expert Input): This stage aims to consider what resources are available for addressing the need. These resources, such as issues arising from the miniuser testing results and the literature review, require a discussion amongst experts (in the domain and/or usability) in order to obtain a broader understanding of the specifics of the prospective domain. Also, it entails garnering more information through conversations with expert evaluators to identify the areas/classification schemes of the usability problems related to the selected domain from the overall results. These areas provide designers and developers with insight into how interfaces can be designed to be effective, efficient and satisfying; they also support more uniform problem description and they can guide expert evaluators in finding real usability problems, thereby facilitating the evaluation process by judging each area and page in the target system.

Development Step Four (D4: Draw Up DSI: data analysis): The aim of this step is to analyse all the data gathered from the previous three. Then, the DSI method will be established (as guidelines or principles) in order to address each area of the selected domain.

\section{RESEARCH METHODOLOGY}

\section{A. Evaluation of the Adaptive Framework to Generate the DSI Adaptive Checklist}

In the first stage, the researchers conducted a literature review on the materials relating to usability and UEMs as well as on the requirements of educational websites. In stage two, a mini-user testing session was conducted through a brief questionnaire that consists of four tasks, which were sent to ten users who are regular educational website users. In stage three, a focus group discussion session was carried out with eight experts in usability and the educational domain (i.e. single and double experts). Cohen's kappa coefficient was used on the same group twice to enable a calculation of the reliability quotient for identifying usability problem areas. In stage four, the researchers analysed the results of the previous three stages and incorporated findings. The intra-observer test-retest using Cohen's kappa yielded a reliability value of 0.8 , representing satisfactory agreement between the two rounds. After that, the usability problem areas were identified to facilitate the process of evaluation and analysis, and to help designers and programmers to identify the areas in their systems that need improvement. Then, the DSI method was established, closely focused on educational websites, taking into an account what is called "learner-centred design". The DSI method was classified according to the usability problem areas, and checklist was developed, as shown in Table 1 in the appendix.

\section{B. Piloting the Adaptive Checklist}

A pilot study was conducted by two independent evaluators. They checked the adaptive checklist by applying it in a real experiment to make sure that there were no spelling or grammatical errors and no ambiguous words or phrases, and that all of the sentences in the adaptive checklist were 
sufficiently clear to be used by the evaluators. A fewer minor improvements were made,

\section{Selection of the Targeted Websites}

The researchers selected free educational websites (Bhargava et al., 2013). The selection process of the websites was criteria-based; 6 aspects were determined and verified for each website to achieve the research aim, and these are: 1) Good interface design,2) Rich functionality, 3) Good representatives of the free educational websites, 4) Not familiar to the users, 5) No changes will occur before and during the actual evaluation, and 6) Completely free educational websites. In order to achieve a high level of quality in this research, the researchers chose three well-known websites in this domain that each has all the aspects mentioned above (skoool, AcademicEarth, BBC KS3bitesize).

\section{Actual Evaluation}

After constructing the DSI checklist, the researchers test it intensively through rigorous validation methods to verify the extent to which it achieves the identified goals, needs and requirements that the adaptive DSI checklist was originally developed to address. It was conducted alongside heuristics evaluation (HE) and user testing (UT). The aim of this process is to collect data ready for analysis (analytically, empirically, and statistically).

Therefore, 8 expert evaluators were recruited to use the adaptive DSI checklist and $\mathrm{HE}$ checklist that had been developed by the researchers to facilitate the evaluation process for both methods. The evaluators had selected from the adaptive DSI checklist the usability areas and the appropriate principles for each website. Then, the actual expert evaluation was conducted and the evaluators evaluated all websites consecutively, rating all the problems they found in a limited time (which was 90 minutes). After that, they were asked to submit their evaluation report, and to give feedback on their own evaluation results. Next, 60 users were recruited for using UT. Before starting the actual evaluation, all users were given a UEM training pack. Each user was given the task scenario sheet and asked to read and then perform one task at a time.

\section{Discussion AND Findings}

The researchers extracted the problems discovered by the three methods from the problems sheet and removed all false positive problems, subjective problems, and duplicated problems during the debriefing session. The problems agreed upon were merged into a unique master problem list (see Table 1, Table 2, and Table 3), and any problems upon which the evaluators disagreed were removed.

\section{TABLE I. TOTAL PROBLEMS Found IN BBC Ks3BITESIZE}

\begin{tabular}{|l|c|l|l|c|}
\hline $\begin{array}{r}\text { Method } \\
\text { Problem type }\end{array}$ & UT & HE & DSI & $\begin{array}{l}\text { Total } \\
\text { problems }\end{array}$ \\
\hline Catastrophic & $0(0 \%)$ & $0(0 \%)$ & $1(100 \%)$ & 1 \\
\hline Major & $2(66 \%)$ & $0(0 \%)$ & $3(100 \%)$ & 3 \\
\hline Minor & $5(100 \%)$ & $0(0 \%)$ & $5(100 \%)$ & 5 \\
\hline Cosmetic & $9(100 \%)$ & $2(22 \%)$ & $2(22 \%)$ & 11 \\
\hline No. problems & $16(80 \%)$ & $2(10 \%)$ & $12(60 \%)$ & 20 \\
\hline
\end{tabular}

TABLE II. TOTAL PROBLEMS FOUND IN SKOOOL

\begin{tabular}{|l|l|l|l|c|}
\hline $\begin{array}{c}\text { Method } \\
\text { Problem type }\end{array}$ & UT & HE & DSI & $\begin{array}{l}\text { Total } \\
\text { problems }\end{array}$ \\
\hline Catastrophic & $1(25 \%)$ & $2(50 \%)$ & $4(100 \%)$ & 4 \\
\hline Major & $3(30 \%)$ & $2(20 \%)$ & $6(89 \%)$ & 7 \\
\hline Minor & $2(29 \%)$ & $3(43 \%)$ & $11(85 \%)$ & 11 \\
\hline Cosmetic & $7(54 \%)$ & $3(23 \%)$ & $12(92 \%)$ & 12 \\
\hline No. of problems & $13(38 \%)$ & $10(29 \%)$ & $33(97 \%)$ & 34 \\
\hline
\end{tabular}

TABLE III. TOTAL PROBLEMS FOUND IN ACADEMIC EARTH

\begin{tabular}{|l|l|l|l|c|}
\hline $\begin{array}{r}\text { Method } \\
\text { Problem type }\end{array}$ & UT & HE & DSI & $\begin{array}{l}\text { Total } \\
\text { problems }\end{array}$ \\
\hline Catastrophic & $0(0 \%)$ & $1(33 \%)$ & $3(100 \%)$ & 3 \\
\hline Major & $3(50 \%)$ & $3(50 \%)$ & $4(66 \%)$ & 6 \\
\hline Minor & $2(17 \%)$ & $7(58 \%)$ & $11(92 \%)$ & 12 \\
\hline Cosmetic & $7(50 \%)$ & $2(14 \%)$ & $11(79 \%)$ & 14 \\
\hline No. of problems & $12(34 \%)$ & $13(37 \%)$ & $29(83 \%)$ & 35 \\
\hline
\end{tabular}

Generally, UT, HE and adaptive DSI checklist revealed different types and numbers of usability problems. One-way ANOVA reveals that there is significant difference between the three methods in terms of discovering usability problems on the whole $(\mathrm{F}=13.447, \mathrm{p}<0.001)$. UT, HE and the adaptive DSI checklist revealed $80 \%, 10 \%$ and $60 \%$ of the real usability problems found in the BBC KS3bitesize website, respectively. One-way ANOVA-Tukey HSD was used and the results show that there is a strongly significant difference amongst the methods in finding usability problems on the BBC KS3bitesize website between HE and UT, where $\mathrm{p}=0.003$. In the Skoool website, UT, HE and the adaptive DSI checklist revealed 38\%, $29 \%$ and $97 \%$ of the found real usability problems, respectively. One-way ANOVA-Tukey HSD was used and the results show that there is a strongly significant difference amongst the methods in finding usability problems in Skoool (as a dependent factor), particular between HE and the adaptive DSI checklist and between the adaptive DSI checklist and UT, where $\mathrm{p}<0.001$. Finally, UT, HE and the adaptive DSI checklist revealed $34 \%, 37 \%$ and $83 \%$ of the found real usability problems in Academic Earth, respectively. One-way ANOVA-Tukey HSD was used and the results show that there is a significant difference amongst the methods in finding usability problems in Academic Earth between HE and UT, where $\mathrm{p}=0.044$. The performance of $\mathrm{HE}$ in discovering real usability problems totally ranged from $10 \%$ to $37 \%$. UT discovered real usability problems ranging from $34 \%$ to $80 \%$, while the adaptive DSI checklist discovered real usability problems ranging from $60 \%$ to $97 \%$. Also, UT and $\mathrm{HE}$ performed better in discovering major, minor and cosmetic real usability problems, but the adaptive DSI checklist was the best in discovering more catastrophic, major, minor and cosmetic real usability problems. Furthermore, 9 unique problems were discovered in all experiments on the three websites through UT (6 in BBC KS3bitesize and 3 in Academic Earth); whereas the remaining UT problems were discovered by the adaptive DSI checklist (although one was discovered by HE). Thus, it can be seen that the adaptive DSI checklist was the best in discovering real problems; UT came second, and HE in third place.

\section{CONClusion AND Future Work}

The main aim of this experiment was to evaluate the adaptive DSI checklist for the educational websites through its 
ability to discover usability problems by comparing its results with usability testing (UT) and heuristic evaluation (HE). The adaptive DSI checklist seemed to guide the evaluators' thoughts in judging the usability of the websites. This finding facilitates decision-making with regard to which of these methods to employ. Also, it addresses the shortcomings of these methods; hence, to avoid wasting money and time an alternative method that is well-developed, context-specific and adaptive to the situation in hand, such as what has been generated here for the educational domain, should be employed. This research contributes to the advancement of knowledge in the HCI field by introducing the adaptive DSI checklist that is specific for evaluating educational systems. In order to consolidate and confirm the findings, future research could include testing the adaptive DSI checklist by applying it, for example, to web-based server applications. Also, we need to further test the adaptive framework by developing an adaptive DSI checklist for different fields, such as e-commerce or news sites.

\section{REFERENCES}

[1] Alias, N. Siraj, S. DeWittD. Attaran, M.and Nordin, A.(2013). Evaluation on the usability of physics module in a secondary school in Malaysia: Students retrospective", The Malaysian Online Journal of Educational Technology, p. 44.

[2] AlRoobaea, R. Al-Badi, A. Mayhew P. (2013a). Generating a Domain Specific Inspection Evaluation Method through an Adaptive Framework: A Comparative Study on Educational Websites. International Journal of Human Computer Interaction (IJHCI), Volume 4, NO. 2.

[3] AlRoobaea, R. Al-Badi, A. Mayhew P. (2013b). Generating a Domain Specific Inspection Evaluation Method through an Adaptive Framework: A Comparative Study on Social Networks. International Journal of Advanced Computer Science and Applications. Volume 4, No. 6.
[4] Bhargava, P., Dhand, S., Lackey, A. E., Pandey, T., Moshiri, M., \&Jambhekar, K. (2013). Radiology Education 2.0-On the Cusp of Change: Part 2. eBooks; File Sharing and Synchronization Tools; Websites/Teaching Files; Reference Management Tools and Note Taking Applications. Academic Radiology, 20(3), 373-381.

[5] Chattratichart, J.and LindgaardG.(2008).A comparative evaluation of heuristic-based usability inspection methods. In CHI, vol. 8, pp. 05-10.

[6] Chen S.and Macredie, R. (2005).The assessment of usability of electronic shopping: A heuristic evaluation. International Journal of Information Management, vol. 25, no. 6, pp. 516-532.

[7] Coursaris,C.and Kim, D. (2011).A meta-analytical review of empirical mobile usability studies. Journal of Usability Studies, vol. 6, no. 3, pp. 117-171.

[8] Guo, Y. Proctor, R.and Salvendy, G. (2011).A conceptual model of the axiomatic usability evaluation method",Human Interface and the Management of Information. Interacting with Information, pp. 93-102.

[9] ISO (1998). ISO 9241-11: Ergonomic Requirements for Office Work with Visual Display Terminals (VDTs): Part 11: Guidance on Usability.

[10] Lindgaard G., and Chattratichart,J. (2007). Usability testing: what have we overlooked?" in Proceedings of the SIGCHI conference on Human Factors in Computing Systems, pp. 1415-1424, ACM.

[11] Mankoff, J. Dey, A. Hsieh, G. Kientz, J. Lederer S.and Ames, M.(2003).Heuristic evaluation of ambient displays", in Proceedings of the SIGCHI Conference on Human Factors in Computing Systems, pp. 169-176, ACM.

[12] Nayebi, F. DesharnaisJ., and Abran, A.(2012).The state of the art of mobile application usability evaluation", in Electrical \& Computer Engineering (CCECE), $201225^{\text {th }}$ IEEE Canadian Conference on, pp. 14, IEEE.

[13] Nielsen, J. (2001). Did poor usability kill e-commerce,in www.useit.com.

[14] Oztekin, A. Kong Z.and Uysal, O. (2010).Uselearn: A novel checklist and usability evaluation method for e-learning systems by criticality metric analysis.International Journal of Industrial Ergonomics, vol. 40, no. 4, pp. 455-469.

[15] Rogers, Y., Sharp, H., and Preece, J. (2007). Interaction design: beyond human-computer interaction. Wiley.

[16] Zaharias P., and Poylymenakou, A. (2009). Developing a usability evaluation method for e-learning applications: Beyond functional usability,Intl. Journal of Human-Computer Interaction, vol. 25,no. 1, p. 75-98. 
Table 1: The adaptive Domain Specific Inspection checklist for evaluating educational system usability

\begin{tabular}{|c|c|}
\hline $\begin{array}{l}\text { Usability } \\
\text { problem area }\end{array}$ & The adaptive Domain Specific Inspection (DSI) checklist \\
\hline \multirow{5}{*}{ User usability } & $\begin{array}{l}\text { Supports modification and progress of evaluation: } \\
\text { ○ Does the system make important keys larger than other keys? } \\
\text { ○ Does the system anticipate the user's next activity correctly? } \\
\text { o Does the system allow the user to initiate actions? } \\
\text { o Does the system provide an overview of the work process that has been completed by the user? }\end{array}$ \\
\hline & $\begin{array}{l}\text { Supports user tasks and avoids difficult concepts: } \\
\text { o Does the system provide constructive, brief, unambiguous descriptions of the task when needed? } \\
\text { o Does the system match the menu structure to the task structure? Can the user distinguish between options and } \\
\text { content on the pages? Are there breadcrumbs to show where the user is and where the user last was? } \\
\text { o Does the system use clear, simple language for questions and answers? } \\
\text { o Does the system provide correct spelling and grammar, and understandable graphic symbols? } \\
\text { o Does the system provide the minimal number of clickable actions, infrequent selection, and infrequent scrolling to } \\
\text { complete one main task? Are lesson pages easy to bookmark? } \\
\circ \text { Is an item visible when it should be hidden from the view, and vice versa? }\end{array}$ \\
\hline & $\begin{array}{l}\text { Feedback and support services: } \\
\text { o Is feedback given at any specific time tailored to the content or problem being studied by the user? } \\
\text { o Does feedback provide the user with meaningful information concerning their current level of achievement within } \\
\text { the program? Is any message of current status related to the user's task } \\
\text { o Does the system program provide the user with opportunities to access extended feedback from instructors through } \\
\text { email and Internet communication? Is adequate FAQ offered? } \\
\text { ODothe performance support tools provided mimic their real-world counterparts? }\end{array}$ \\
\hline & $\begin{array}{l}\text { Error Prevention: } \\
\text { o Do error messages prevent potential errors from happening? } \\
\text { o Does the system provide solutions that help the user recover from errors, such as providing undo and redo } \\
\text { features? } \\
\text { o Can errors be averted or minimized when possible? }\end{array}$ \\
\hline & $\begin{array}{l}\text { Easy to remember: } \\
\text { Is a casual user able to return to using the system after some period without having to learn everything all over } \\
\text { again? Are all functions and information well presented to support memorability? }\end{array}$ \\
\hline \multirow{3}{*}{$\begin{array}{l}\text { Motivational } \\
\text { factors }\end{array}$} & $\begin{array}{l}\text { Supports leaner curiosity: } \\
\text { O Does the system support the user's cognitive curiosity through surprises, paradoxes and humour, and does it deal } \\
\text { with topics that are already of interest to the user? }\end{array}$ \\
\hline & $\begin{array}{l}\text { Learning content design and Attractive screen design: } \\
\text { o Are the vocabulary and the terminology used appropriate and presented with good background, giving suitable } \\
\text { examples? } \\
\text { o Is the organization of the content pieces and learning objects suitable for achieving the primary goals of the } \\
\text { system? } \\
\text { o Are similar learning objects organized in a similar style? } \\
\text { o Is the screen layout efficient and visually pleasing (it should appear simple, i.e., uncluttered, readable and } \\
\text { memorable)? } \\
\text { o Are the font choices, colours and sizes consistent with good user screen design? }\end{array}$ \\
\hline & $\begin{array}{l}\text { Motivation to learn: } \\
\text { o Does the system use e-stories, simulations, discussion messages, role-playing and activities to gain the attention } \\
\text { and to maintain the motivation of the user to learn more? } \\
\text { o Does the system provide the user with frequent and varied learning activities that increase learning success? } \\
\text { o Are the user's actions rewarded by audio, video, text or animations, and are the rewards meaningful? } \\
\text { Is the system easy to learn but hard to master? Is the system paced to apply pressure but not frustrate the user? } \\
\text { Does the difficulty level vary so that users are given greater challenges as they develop mastery? } \\
\text { O Is the user's fatigue minimized by varying the activities and the difficulty levels during a learning session? }\end{array}$ \\
\hline \multirow[t]{2}{*}{$\begin{array}{l}\text { Content } \\
\text { information } \\
\text { and process } \\
\text { orientation }\end{array}$} & $\begin{array}{l}\text { Relevant, correct and adequate information: } \\
\text { o Does the system display only information that is relevant to its purposes? } \\
\text { o Does the system update the content constantly? } \\
\text { o Does the system display only the available lesson, and is the content suitable to the page length? } \\
\text { o Does the system provide concise and non-repetitive information? } \\
\text { o Does the system offer an amount of information that isappropriate to the page length, and is all the text of a } \\
\text { viewable/readable size? }\end{array}$ \\
\hline & $\begin{array}{l}\text { Reliability and Validity: } \\
\text { O Is there a link provided to the homepage? Was the system was built by a reliable institution? } \\
\text { o Are reliability, stability and continuity of learning in the system guaranteed? }\end{array}$ \\
\hline
\end{tabular}




\begin{tabular}{|c|c|}
\hline & $\begin{array}{c}\text { Privacy and Security: } \\
\text { o Are sensitive areas protected by passwords and an SSL protocol (e.g., VeriSign }{ }^{\mathrm{TM})} \text { against hackers? }\end{array}$ \\
\hline \multirow{6}{*}{$\begin{array}{l}\text { Learning } \\
\text { process }\end{array}$} & $\begin{array}{l}\text { Assessment: } \\
\text { o Does the system include self-assessment for each module, whether in audio, video or text, and does it keep a record } \\
\text { of progress? } \\
\text { o Does the system provide sufficient feedback (audio, video) to the user in order to provide corrective directions? } \\
\text { o Does the system provide the instructor with the user's evaluation and tracking reports? }\end{array}$ \\
\hline & $\begin{array}{l}\text { Interactivity: } \\
\text { o Does the user become engaged with the system program through activities that are challenging? Is the presentation } \\
\text { of the lessons designed to promote engagement? } \\
\text { o Is the user able to respond to the program at leisure? } \\
\text { o Does learning become easier with an interactive approach, wherein users quickly learn how best to respond to the } \\
\text { program? Does the user gain in confidence by so doing? } \\
\text { oDoes the user have confidence that the system is interacting and operating in the way it was designed to? }\end{array}$ \\
\hline & $\begin{array}{l}\text { Evokes mental images for the users: } \\
\text { o Does the system allow the user to use their imagination, in a way that enhances their comprehension? } \\
\text { ○ Does the system appeal to the imagination and does it encourage recognition in order for the user to create unique } \\
\text { interpretations of the characters or contexts? } \\
\text { o Is the user interested in the system characters because they are drawn from the user's own culture? }\end{array}$ \\
\hline & $\begin{array}{l}\text { Resources: } \\
\text { o Does the system provide access to a wide range of resources (e.g., examples and real data archives) appropriate to } \\
\text { the learning context? } \\
\text { oIf the system includes links to external www. Or to intranet resources, are the links kept up to date? }\end{array}$ \\
\hline & $\begin{array}{l}\text { Learning management: } \\
\text { o Can the user manage all the activities pertaining to the learning program with ease? Can the user clearly } \\
\text { understand everything, and perceive options for additional guidance (chat, edit, add, seek instruction or other forms } \\
\text { of assistance) when needed? Are all control items logically labelled and grouped in a control panel? } \\
\text { o Are the lessons easy to upload, download, share, retrieve and organise? Do the lessons support various learning } \\
\text { styles, and do they support synchronous and asynchronous modes. }\end{array}$ \\
\hline & $\begin{array}{l}\text { Learnability: } \\
\text { oIs the system designed such that the user finds it easy to learn how to use? }\end{array}$ \\
\hline \multirow{4}{*}{$\begin{array}{l}\text { Design and } \\
\text { media usability }\end{array}$} & $\begin{array}{l}\text { Multimedia representations: } \\
\text { o Does multimedia help the user in all aspects to learn interactively by playing videos, audio files and audio mock } \\
\text { tests, and does it make learning enjoyable? } \\
\text { o Does the system include sound and visual effects? Do these effects provide meaningful feedback or hints, and do } \\
\text { they stir particular emotions? } \\
\text { o Does the system include surprises, humour and interesting representations for the user, and does it avoid } \\
\text { unnecessary multimedia representations that could confuse a user who has just started to work with the system? } \\
\text { o Is the user allowed to skip non-playable and frequently repeated content in videos or learning games? } \\
\text { o Is the user allowed to customize video and audio settings, and to adjust the difficulty level? }\end{array}$ \\
\hline & $\begin{array}{l}\text { Accessibility and compatibility of hardware devices: } \\
\text { o Is the system compatible with various platforms and hardware? Are its features adaptable to individual user } \\
\text { preferences? } \\
\text { o Do potential users have all the necessary computer skills to use the application? (There should be consistency } \\
\text { between the motor effort and skills required by the hardware and the developmental stage of the learner audience.) } \\
\text { o Are all input devices/buttons that have no functionality disabled to prevent user-input errors? } \\
\text { o Are the lessons accessible to users with physical impairments, and are their contents available in various } \\
\text { languages? }\end{array}$ \\
\hline & $\begin{array}{l}\text { Functionality: } \\
\text { Is all the necessary functionality of the system available without having to leave the site, and does it work } \\
\text { correctly? } \\
\text { o Is all functionality clearly labelled, and does it facilitate easy task completion? Is the system status of each task } \\
\text { clear on all pages? }\end{array}$ \\
\hline & $\begin{array}{l}\text { Navigation and Visual clarity: } \\
\text { o Are navigation objects and tools kept in particular, clearly defined positions, and are they of an adequately viewable } \\
\text { size? } \\
\text { o Is unnecessary animation and Flash avoided? } \\
\text { o Is content logically structured in different sections and levels with enough space between the individual items? Are } \\
\text { the colours and graphics used suitable for promoting navigation? } \\
\text { o Are the menus understandable and straightforward, and are the items logically grouped and labelled? Do all } \\
\text { buttons, links and features have a 'mouseover' or pop-up window that provides meaningful feedback? } \\
\text { o Is a site map and/or a table of contents available, as well as a calendar? } \\
\text { o Is the site navigation consistent, and is the search engine accurate? }\end{array}$ \\
\hline
\end{tabular}




\begin{tabular}{|l|l|}
\hline & $\begin{array}{l}\text { o Is the user's current position in the system clearly labelled, and are adequate 'back buttons'(to previous pages) } \\
\text { provided? Can the user clearly identify where to start on the system's Homepage? } \\
\text { o Is the need for scrolling down a page kept to a minimum? } \\
\text { o Are all functions, buttons and links labelled meaningfully, and are their intended functionalities clear? }\end{array}$ \\
\hline
\end{tabular}

